\title{
Automated Ham Quality Classification Using Ensemble Unsupervised Mapping Models
}

\author{
Bruno Baruque ${ }^{1}$, Emilio Corchado ${ }^{1}$, Hujun $_{\text {Yin }^{2}}$, Jordi Rovira ${ }^{3}$, and Javier González ${ }^{3}$ \\ ${ }^{1}$ Department of Civil Engineering. University of Burgos, Spain. \\ bbaruque@ubu.es, escorchado@ubu.es \\ ${ }^{2}$ School of Electrical and Electronic Engineering. University of Manchester, UK \\ h.yin@manchester.ac.uk \\ ${ }^{3}$ Department of Biotechnology and Food Science, University of Burgos, Spain \\ jrovira@ubu.es, javigonzalez77@hotmail.com
}

\begin{abstract}
This multidisciplinary study focuses on the application and comparison of several topology preserving mapping models upgraded with some classifier ensemble and boosting techniques in order to improve those visualization capabilities. The aim is to test their suitability for classification purposes in the field of food industry and more in particular in the case of dry cured ham. The data is obtained from an electronic device able to emulate a sensory olfative taste of ham samples. Then the data is classified using the previously mentioned techniques in order to detect which batches have an anomalous smelt (acidity, rancidity and different type of taints) in an automated way.
\end{abstract}

Keywords: topology preserving mappings, ensembles, electronic nose, food industry.

\section{Introduction}

Nowadays, the consumer's trust is very important when you want to consolidate and situate a product into the market. Dry cured ham is a product typically consumed in the Iberian Peninsula which has a high popular acceptation. We understand for "Serrano Ham" a ham salted and dried during more than 210 days and that can be displayed boned or deboned. In this type of product, during the storage period come about the development of rancidity and acid odours; most of then may increase significantly because of proteolysis and lipid oxidation [1]. It is important to find easy, quick and cheap techniques that allow, through the measure of simple parameters, to evaluate the quality of these products before the consumers purchase.

In order to help food industry analysis to support the subjective decisions of human testers, several recently appeared devices designed especially for that purpose can be used. The disadvantage with this alternative type of testing is that what humans interpret as tastes and smells, machines interpret as (usually complicated) numeric measures. Our objective is devising a system capable of interpreting the analyses 
made by an electronic device and making the results of those analyses more easily understandable for human experts.

Topology preserving models are mainly used for data visualization and inspection which is an interesting feature when assisting human in classification tasks such as the one presented in this work. Other features are pattern recognition and automated classification inherent to many of all the unsupervised learning techniques, which are especially useful in the present application. These models are given enhanced stability in this study with the use of ensemble techniques.

A combination of an electronic device for the analysis of volatile compounds (referred as "electronic nose" in the rest of the paper) and topology preserving mapping algorithms upgraded by the use of ensemble meta-algorithms is used to analyse a wide variety of samples of "Serrano" Ham to verify if hams with different olfative characteristics could be easily and reliably discriminated by this procedure.

\section{Preliminary Ham Samples Analysis}

\subsection{Case Study Description}

The objective of this study is to test the suitability of the combination of an "electronic nose" (or e-nose), used to measure the odour characteristics of the ham samples; with unsupervised learning data analysis and visualization models for a simple and reliable ham testing and classification. For that reason, this study has been conducted using several hams of different qualities and origins within the limits of the Spanish territory. The dataset used consists on measures obtained from several brands of seven types of Spanish ham, available in the Spanish market. The samples analysed included also some tainted and/or too rancid/acid samples. The tainted samples were randomly taken from among all the different quality types and origins of hams. The commercial brands from where the samples were extracted are not taken into account in this study.

\subsection{The Use of the Electronic Nose for Odour Recognition}

The main steps of odour recognition can be summarized as follows:

1. Heating the sample for a certain time generates volatile compounds in the headspace of the vial containing the sample.

2. The gas phase is transferred to a detection device which reacts to the presence of molecules.

3. The difference in sensor reactions is revealed using different statistical calculation techniques to classify the odours. The measures taken by each sensor are separated and stored in a simple database for further study.

In this study the analyses are performed by an Electronic Nose $\alpha \mathrm{FOX} 4000$ (AlfaMOS, Toulouse, France) with a sensor array of 18 metal oxide sensors. The e-nose takes dates each 0.5 seconds during 120 seconds of acquisition time and 600 seconds of acquisition delay. Only the highest value obtained for each sensor is stored in the database used for further analysis. 


\section{Competitive Learning Ensembles}

\subsection{The SOM and ViSOM Algorithms}

The Self-Organizing Map (SOM) algorithm [2] and the Visualization Induced SelfOrganizing Map (ViSOM) [3] are different types of Topology Preserving Mappings. This is a family of techniques with a common target: to provide a low dimensional representation of multi-dimensional datasets while preserving the topological properties of the input space. The SOM algorithm is based on a type of unsupervised learning called competitive learning; an adaptive process in which the neurons in a neural network gradually become sensitive to different input categories, sets of samples in a specific domain of the input space [4].

One interesting extension of this algorithm is the ViSOM proposed to directly preserve the local distance information on the map, along with the topology. The ViSOM constrains the lateral contraction forces between neurons and hence regularises the interneuron distances so that distances between neurons in the data space are in proportion to those in the input space [5].

The difference between the SOM and the ViSOM hence lies in the update of the weights of the neighbours of the winner neuron as can be seen from Eqs (1) and (2).

Update of neighbourhood neurons in SOM:

$$
w_{k}(t+1)=w_{k}(t)+\alpha(t) \eta(v, k, t)\left(x(t)-w_{v}(t)\right)
$$

Update of neighbourhood neurons in ViSOM:

$$
w_{k}(t+1)=w_{k}(t)+\alpha(t) \eta(v, k, t)\left(\left[x(t)-w_{v}(t)\right]+\left[w_{v}(t)-w_{k}(t)\right]\left(\frac{d_{v k}}{\Delta_{v k} \lambda}-1\right)\right)
$$

where $w_{v}$ is the winning neuron, $\alpha$ the learning rate of the algorithm, $\eta(v, k, t)$ is the neighbourhood function where $v$ represents the position of the winning neuron in the lattice and $k$ the positions of the neurons in the neighbourhood of this one, $x$ is the input to the network and $\lambda$ is a "resolution" parameter, $d_{v k}$ and $\Delta_{v k}$ are the distances between the neurons in the data space and in the map space respectively.

Both models can be adapted for classification of new samples using a semisupervised procedure [6].

\subsection{Ensembles of Topology Preserving Maps}

The ultimate goal of constructing an ensemble is to improve the performance obtained of a single working unit. When talking about classification it is generally accepted that the sets of patterns misclassified by the different classifiers would not necessarily overlap. This suggests that different classifier designs potentially offer complementary information about the patterns to be classified and could be harnessed to improve the performance of the selected classifier [7]. Many ensemble models and theories have been developed in the previous years that range from a 
quite simple technique like Bagging [8] or AdaBoost [9] to the more sophisticated ones such as LPBoost [10] or and many other variants. These techniques have been mainly applied to models designed specifically for classification, specially supervised classifiers [11].

Bagging and AdaBoosting. Boosting meta-algorithms consist on training a simple classifier in several stages by incrementally adding new capacities to the current learned function.

Bagging (or bootstrap aggregating) [8] consists on training each of the classifiers composing the ensemble separately using a different subset of the main training dataset. This is accomplished by using re-sampling with replacement over the training set. The classification results are obtained by (weighted or majority) voting among its composing classifiers. The technique provides the ensemble with a balance between variability and similarity.

AdaBoost [9] is another algorithm that gives as a result an ensemble but its training method is a bit more developed. The difference with the previous method is that it is taken into accounts which of the training samples are not correctly classified by the current classifier. That way, the ensemble concentrates in the samples that are harder to classify, improving its learning capabilities. The final output of the ensemble is a weighted vote of all the classifiers composing it. Schapire and Freund [11] proposed two slightly different versions of the algorithm. In this work AdaBoost.M2 is used, as it is recommended for datasets with more than two different classes. Although this meta-algorithm has been previously used for supervised classification [12], a short number of studies [13], [14], [15] involves unsupervised one such as in the present work.

Combining Ensemble Results. It must be taken into account that the models used in this research were originally designed as visualization tools. Constructing classical ensembles can be considered as a good option when trying to boost their classification capabilities, stabilizing its learning algorithm and avoiding overfitting; but when dealing with its visualization feature an ensemble is not directly displayable [16], [15]. To overcome this problem, some "ensemble combination" algorithms have been devised [17] in order to obtain a unique network that somehow represents the information contained in the different networks composing the ensemble. Our objective is that this "combination" unites good classification accuracy with truthful representation of data for visual inspection.

The procedure is the same for all the tests involving this combination of networks. A simple n-fold cross-validation is used in order to employ all data available for training and testing the model and having several executions to calculate an average of its performance. In each step of the cross-validation, first an ensemble of networks must be obtained. The way in which the ensemble is trained does not affect the system in which the combination is computed. In the case of this study, this has been done using the bagging or the adaboost meta-algorithm. Then the computation of the combination is performed. Finally both, the ensemble and the combination generated from it are tested employing the test fold. In order to obtain the similarity needed to make networks comparable to combine them, the networks of the same ensemble are 
initialized to the inter-neural weights obtained in the training of the previous network.

The different options studied for combining the network of the ensemble into a single network, summarizing its main characteristics are described in the following paragraphs. Further details can be found on [17], [18].

Ensemble Combination 1: Fusion [19] involves comparing the networks neuron by neuron in the input space. This implies that all the networks in the ensemble must have the same size. First, it searches for the neurons that are closer in the input space (selecting only one neuron in each network of the ensemble) then it "fuses" them to obtain the final neuron in the "fused" map. This process is repeated until all the neurons have been fused. In this case, a re-labelling of the neurons of the fused network, employing again the training dataset, is done in order to get a clear visualization of the map.

Ensemble Combination 2: Superposition [20] consists on "superposing" the maps formed by the networks composing the ensemble into a final map, on a neuron by neuron comparison (as is done in fusion). Note that the weights of each network in the ensemble are initialized in a way that makes the neurons in the same position of two (or more) networks comparable. On the contrary of fusion, this technique relies in the way the networks are trained to compute an acceptable summary, so the calculations on the combination phase are less time-consuming. The method ensures that the resultant "summarized" or "superposed" map represents visually what the majority of the maps composing the ensemble represent in a neuron-by-neuron basis. When using the resultant "superposed" map for classification purposes it returns the class represented by the neuron that is activated when the new sample is presented to the network.

Ensemble Combination 3: Superposition + Re-labelling [20] has two main phases. The first one is the superposition explained before. The second one consists of testing which class actually recognizes better each neuron after the superposition, instead of relying on the recognition of the neurons in the same position done previously in the individual testing of each of the ensemble networks. So, after the superposition phase, the same dataset used for training is presented to the resultant network of the superposition to check which class is more consistently recognized by each neuron. Usually less number of neurons responds to this re-labelling, giving as a result a more compact map.

\section{Experiments and Results}

After having been recorded by the electronic nose, the measures of the 18 sensors are stored for each sample of ham cut in a database along with its corresponding human tester cataloguing. This cataloguing is normally more detailed, but for this initial study, it has been simplified to only three possible values: "non-spoiled", "rancid/acid" and "tainted". Thus, our final dataset consists in a total of 176 samples of ham, each of them composed of 18 different variables measured over it and 3 possible classes. 


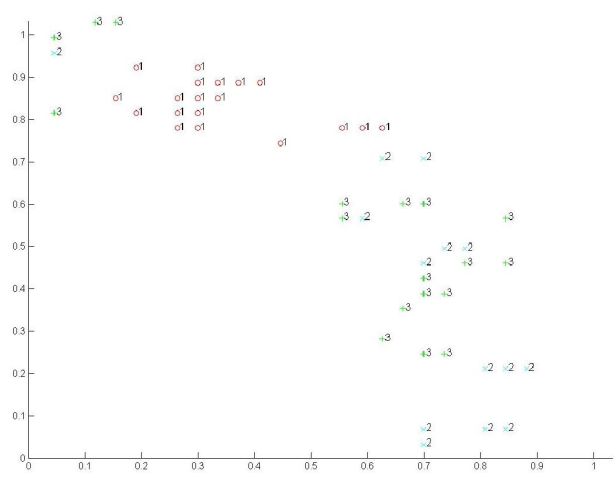

a) Single ViSOM map

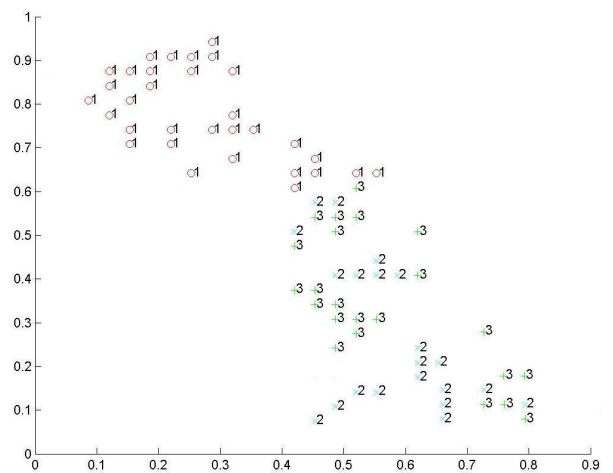

c) Superposition + Re-labelling combination of a ViSOM ensemble

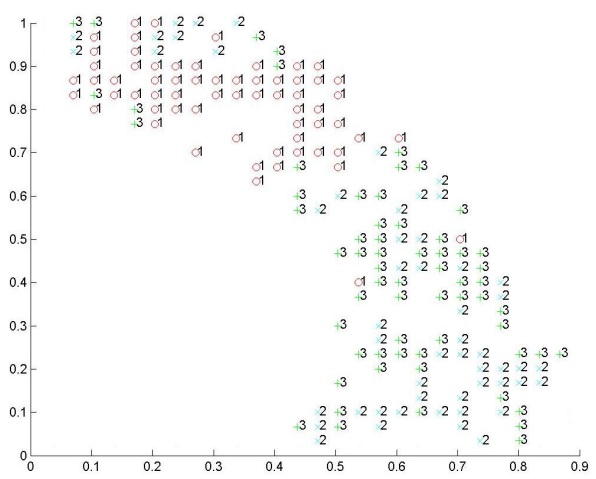

b) Superposition combination of a ViSOM ensemble

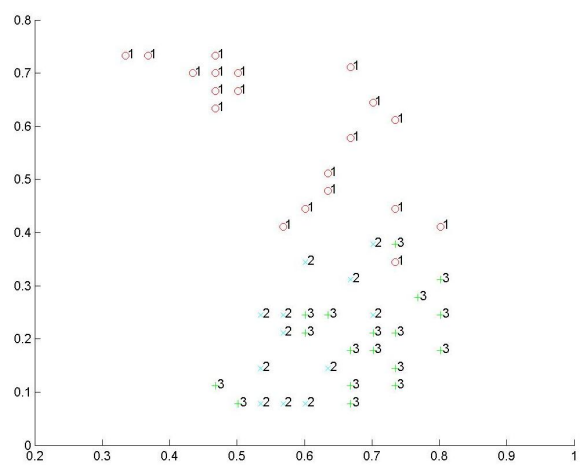

d) Fusion combination of a VISOM ensemble

Fig. 1. The four images (a), (b), (c) and (d) are obtained from VISOM models of a size of 30x30. The 'non-spoiled' samples are marked with a 1 (top of the images), 'acid/rancid' are marked as 2 and 'tainted' samples with 3 .

Regarding to the visualization capabilities of the two topology preserving mapping models employed, the ViSOM is the one that obtains better results in this aspect, as it is specially devised for enhancing visualization. The use of ensemble and combination techniques can certainly improve the quality of the final map obtained, gaining in stability during the training phase and obtaining much less sparse maps, as the final representation can include all the responding neurons of the maps composing the ensemble. Among the ensemble combination methods, the Superposition $+\mathrm{Re}-$ labelling seems to capture better the internal structure of the dataset, obtaining a similar image as a single network. This can be observed in Fig. 1 a) to d).

What is interesting in this kind of analysis is that you get visual (and therefore immediate) information of the level of quality of a sample within its main group. In Fig. 2 neurons identified with 7, 10 and 12 are activated by the highest quality samples. Those identified 11 are activated by standard quality samples. Neurons marked with 2 and 3 are activated by samples that were previously classified as standard, but were not conserved properly and started to get spoiled. Neurons marked 
with 1, 6, 8 and 9 are activated by 'acid/rancid' samples. Neurons marked with 4, 5 and 14 are the ones that are activated by definitively 'tainted' samples. As it can be seen in Fig 2 neurons recognising 'non-spoiled' samples are all situated at the top of the image (in Fig 1(c) marked with 1s). Within this group, the neurons activated by highest quality samples are above the ones activated by the standard quality ones, which appear closer to 'acid/rancid' group. The samples that were previously considered to be of standard quality (marked with 2 and 3) and got rancid are now recognized by neurons situated among the spoiled samples recognizing neurons.

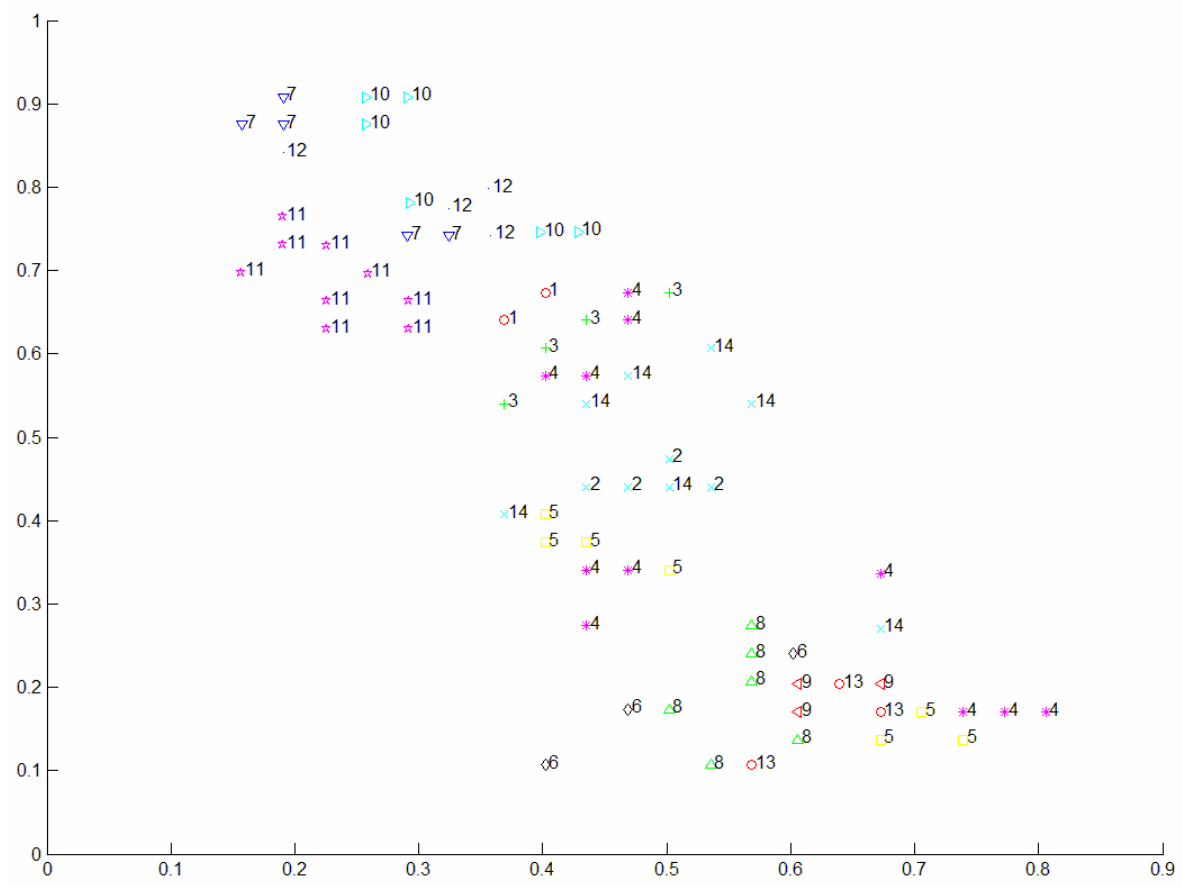

Fig. 2. The image is obtained form the same ensemble combination as Fig 1 (c), but using a more expressive classification on data samples, indicating different levels of quality among the previously described 3 groups

Table 1. Model classification accuracy using a 10 fold cross-validation over the dataset. The ensembles are all composed of 10 networks. The training was done employing the bagging meta-algorithm.

\begin{tabular}{l|ccccc}
\hline \multicolumn{1}{c|}{ Model } & $\begin{array}{c}\text { Best Single } \\
\text { Netwk. }\end{array}$ & $\begin{array}{c}\text { Ensemble } \\
\text { (weighted voting) }\end{array}$ & Superp. & $\begin{array}{c}\text { Superp. + Re- } \\
\text { Labelling }\end{array}$ & Fusion \\
\hline SOM (10x10) & $68.4 \%$ & $84.1 \%$ & $73 \%$ & $69 \%$ & $78.4 \%$ \\
SOM (20x20) & $49.5 \%$ & $78.7 \%$ & $62.2 \%$ & $57.7 \%$ & $59.3 \%$ \\
SOM (30x30) & $40.8 \%$ & $67.2 \%$ & $49.1 \%$ & $52.6 \%$ & $52.1 \%$ \\
ViSOM (20x20) & $78 \%$ & $90 \%$ & $87.1 \%$ & $83.9 \%$ & $79.7 \%$ \\
ViSOM (30x30) & $69.1 \%$ & $82.8 \%$ & $81.5 \%$ & $61.7 \%$ & $60.4 \%$ \\
ViSOM (40x40) & $62.5 \%$ & $86.9 \%$ & $52.7 \%$ & $70.4 \%$ & $71.3 \%$ \\
\hline
\end{tabular}


Regarding the automated classification of samples, the SOM and ViSOM behave in a similar way. As it was expected, and can be seen both in Tables 1 and 2, the ensemble of maps obtains a much higher accuracy than its homologue single and combined networks. The three combination models are much more dependent of individual performances, so they obtain much lower results than the ensemble, but still significantly better classification rate than the single model. Adaboost obtains better results than Bagging in most of the cases, although is not a very significant difference, as can be seen comparing results of Table 1 and Table 2.

Table 2. Model classification accuracy using a 10 fold cross-validation over the dataset. The ensembles are all composed of 10 networks. The training was done employing the adaboost.m2 meta-algorithm.

\begin{tabular}{l|ccccc}
\hline \multicolumn{1}{c|}{ Model } & $\begin{array}{c}\text { Best Single } \\
\text { Netwk. }\end{array}$ & $\begin{array}{c}\text { Ensemble } \\
\text { (weighted voting) }\end{array}$ & Superp. & $\begin{array}{c}\text { Superp. + Re- } \\
\text { Labelling }\end{array}$ & Fusion \\
\hline SOM $(10 \times 10)$ & $70.2 \%$ & $82.9 \%$ & $71.7 \%$ & $73.4 \%$ & $78 \%$ \\
SOM $(20 \times 20)$ & $47.8 \%$ & $78.3 \%$ & $62.6 \%$ & $61.7 \%$ & $62.6 \%$ \\
SOM $(30 \times 30)$ & $38 \%$ & $66.7 \%$ & $58.7 \%$ & $49.4 \%$ & $51.7 \%$ \\
ViSOM $(20 \times 20)$ & $80.9 \%$ & $91.1 \%$ & $81.1 \%$ & $82 \%$ & $84.6 \%$ \\
ViSOM $(30 \times 30)$ & $68 \%$ & $90 \%$ & $72.1 \%$ & $70.9 \%$ & $75.5 \%$ \\
ViSOM $(40 \times 40)$ & $64.9 \%$ & $85.6 \%$ & $75.8 \%$ & $66.3 \%$ & $71 \%$ \\
\hline
\end{tabular}

\section{Conclusions}

In the present work a viable way of automated ham testing for quality based on ensembles of topology preserving maps is presented.

With the appropriate training and use of boosting techniques the classification can be very simple to the human expert, as the reliability of the classification can be inherently deduced from the position of the new sample in the map. For example, if the sample under test activates a neuron in a region of the map clearly identified as "nonspoiled" it can be concluded that the sample is definitively tainted. If the sample is represented in a region identified as "non-spoiled" but very close to the "acid/rancid" region it can be concluded that the sample is not as definitively "non-spoiled" as the previous one and will probably be a "non-spoiled" sample with a "rancid" scent.

This visual analysis can be complemented with the classification capabilities of the model that can achieve more than $90 \%$ of accuracy in explicit classification. Note that this measure is calculated using a hard discrimination, while the model is capable of giving a richer interpretation of the classification, providing a percentage of confidence in the inclusion of the sample into one class or another.

Concerning to future work, there are several methods that are regarded as possibly very useful in the present case study and will be tested. Some of them are more evolved boosting algorithms and some method for feature selection to reduce the dimensionality of the dataset in first instance. Also more refined accuracy measurements could be used to determine more exactly the classification capabilities of the models.

Acknowledgments. This research has been supported by the MCyT project TIN200407033. 


\section{References}

1. Monahan, R.L., Brunton, N.P., Cronin, D.A., Durcan, R.: Determination of Hexanal in Cooked Turkey Using Solid Phase Microextraction (Spme)/Gc. In: 44th International Congress of Meat science and Technology (ICoMST), vol. 1, pp. 586-587 (1998)

2. Kohonen, T.: The Self-Organizing Map. Neurocomputing 21, 1-6 (1998)

3. Yin, H.: Data Visualisation and Manifold Mapping Using the Visom. Neural Networks 15, 1005-1016 (2002)

4. Kohonen, T., Lehtio, P., Rovamo, J., Hyvarinen, J., Bry, K., Vainio, L.: A Principle of Neural Associative Memory. Neuroscience 2, 1065-1076 (1977)

5. Yin, H.: Visom - a Novel Method for Multivariate Data Projection and Structure Visualization. Neural Networks, IEEE Transactions on 13, 237-243 (2002)

6. Kraaijveld, M.A., Mao, J., Jain, A.K.: A Nonlinear Projection Method Based on Kohonen's Topology Preserving Maps. Neural Networks, IEEE Transactions on 6, 548$559(1995)$

7. Heskes, T.: Balancing between Bagging and Bumping. In: Advances in Neural Information Processing Systems 9 - Proceedings of the 1996 Conference, vol. 9, pp. 466472 (1997)

8. Breiman, L.: Bagging Predictors. Machine Learning 24, 123-140 (1996)

9. Freund, Y., Schapire, R.E.: A Decision-Theoretic Generalization of on-Line Learning and an Application to Boosting. Journal of Computer and System Sciences 55, 119-139 (1997)

10. Demiriz, A., Bennett, K.P., Shawe-Taylor, J.: Linear Programming Boosting Via Column Generation. Machine Learning 46, 225-254 (2002)

11. Freund, Y., Schapire, R.E.: Experiments with a New Boosting Algorithm. In: International Conference on Machine Learning, pp. 148-156 (1996)

12. Schwenk, H., Bengio, Y.: Boosting Neural Networks. Neural Computation. 12, 1869-1887 (2000)

13. Gabrys, B., Baruque, B., Corchado, E.: Outlier Resistant PCA Ensembles. In: Gabrys, B., Howlett, R.J., Jain, L.C. (eds.) KES 2006. LNCS (LNAI), vol. 4253, pp. 432-440. Springer, Heidelberg (2006)

14. Corchado, E., Baruque, B., Gabrys, B.: Maximum Likelihood Topology Preserving Ensembles. In: Corchado, E., Yin, H., Botti, V., Fyfe, C. (eds.) IDEAL 2006. LNCS, vol. 4224, pp. 1434-1442. Springer, Heidelberg (2006)

15. Petrakieva, L., Fyfe, C.: Bagging and Bumping Self-Organising Maps. Computing and Information Systems (2003)

16. Kaski, S.: Data Exploration Using Self-Organizing Maps. Department of Computer Science and Engineering. Helsinki University of Technology. Espoo, Finland (1997)

17. Baruque, B., Corchado, E., Yin, H.: ViSOM Ensembles for Visualization and Classification. In: International Work Conference on Artificial Neural Networks, San Sebastián, Spain, Springer, Heidelberg (2007) (in press)

18. Baruque, B., Corchado, E., Yin, H.: Boosting Unsupervised Competitive Learning Ensembles. In: International Conference of Neural Network (ICANN 2007) (2007)

19. Georgakis, A., Li, H., Gordan, M.: An Ensemble of SOM Networks for Document Organization and Retrieval. In: Int. Conf. on Adaptive Knowledge Representation and Reasoning (AKRR'05), p. 6 (2005)

20. Baruque, B., Corchado, E., Yin, H.: VISOM Ensembles for Visualization and Classification. In: International Work Conference on Artificial Neural Networks (IWANN'07), San Sebastián, Spain, Springer, Heidelberg (2007) (in press) 